\title{
Investigating $\pi^{-}$production in central ${ }^{7} \mathrm{Be}+{ }^{9} \mathrm{Be}$ and ${ }^{40} \mathrm{Ar}+{ }^{45} \mathrm{Sc}$ collisions at CERN SPS energies with PYTHIA8/Angantyr
}

\author{
Khaled Abdel-Waged ${ }^{1,2, a_{(D}}$ \\ ${ }^{1}$ Physics Department, Faculty of Applied Science, Umm Al-Qura university, Makkah 21421, Saudi Arabia \\ ${ }^{2}$ CERN, Geneva, Switzerland
}

Received: 10 October 2021 / Accepted: 10 January 2022 / Published online: 24 January 2022

(C) The Author(s) 2022

\begin{abstract}
We study pion production in proton-proton (pp) and in central ${ }^{7} \mathrm{Be}+{ }^{9} \mathrm{Be}$ and ${ }^{40} \mathrm{Ar}+{ }^{45} \mathrm{Sc}$ collisions at CERN super proton synchrotron (SPS) energies within the Angantyr model, whose pp dynamics are modeled by PYTHIA 8.303 event generator. We have tuned the parameters of the default multi-partonic interaction (MPI) mechanism in PYTHIA 8.303 to describe simultaneously the measured rapidity and transverse momentum distributions of pions in inelastic pp-collisions in the beam momentum range from 20 to $158 \mathrm{GeV} / \mathrm{c}$. We explore the influence of the standard Lund fragmentation and thermal string models, on the $\pi^{-}$ observables, mainly from light(er) ion beams at CERN SPS energies. The role of primary hadron rescattering $(\mathrm{HadSc})$ is also discussed. We find that the PYTHIA 8.303/Angantyr model employing both pp-tuned and thermal-string model with HadSc is able to reproduce the observed $\pi^{-}$yields in the light(er) ion beams at the whole CERN SPS energies.
\end{abstract}

\section{Introduction}

The production mechanisms of hadrons from nucleusnucleus (AA) collisions may shed light on the formation of a new state of matter - the quark-gluon-plasma (QGP). It is conceived that a QGP can be partly formed in high multiplicity proton-proton (pp) reactions at the same energy per nucleon. Several features such as strangeness enhancement [1], formation of a ridge [2] and $J / \Psi$ suppression [3] are observed in both pp and central heavy-ion collisions, and have been interpreted as a QGP signature.

PYTHIA event generator [4] is commonly used for the description of pp-collisions from the $\mathrm{GeV}$ to $\mathrm{TeV}$ energy range. The code is incorporated in many microscopic transport models, such as UrQMD [5], HIJING [6], and AMPT [7], for generation of full exclusive events of AA-collisions and

\footnotetext{
a e-mail: kamabdellatif@uqu.edu.sa (corresponding author)
}

frequently applied in comparisons to experimental data from NA61/SHINENA61 [8-12], STAR [13] and ALICE [14] collaborations. These event generators simulate the dynamics of hard and soft processes from high momentum transfer ( $Q \geq 2 \mathrm{GeV}$ ), where perturbation theory is applicable, down to scales, where one must rely on string and hadronic models.

It is demonstrated in Refs. $[10,12]$ that the final multiplicities of $\pi^{-}$in central ${ }^{7} \mathrm{Be}+{ }^{9} \mathrm{Be}$ and ${ }^{40} \mathrm{Ar}+{ }^{45} \mathrm{Sc}$ collisions are not reproduced by the well tested models of hadronization adopted in the Monte Carlo (MC) event generators such as UrQMD [5], HIJING [6] and AMPT [7]. The experimental facts raise the question of whether the description of $\pi^{-}$high multiplicity in central light(er) is due to finding the right settings of hadronization models, or whether it requires invoking a QGP-like-state in MC generators. An excellent example of the latter approach is the core-corona model [15] implemented in the EPOS MC event generator [16]. It is assumed that a high density thermalized matter (core) hadronizes like a plasma, while the outer lower density corona does not [15]. The EPOS (version 1.99) model (that with core production), however, does not also provide a consistent description of the measured pion yield in central $\mathrm{Ar}+\mathrm{Sc}$ at SPS energies [12].

In this work, we attempt a further investigation of the recent NA61/SHINE data $[10,12]$, of negative pions in central ${ }^{7} \mathrm{Be}+{ }^{9} \mathrm{Be}$ and ${ }^{40} \mathrm{Ar}+{ }^{45} \mathrm{Sc}$ collisions at beam momentum of $13 \mathrm{~A}, 19 \mathrm{~A}, 30 \mathrm{~A}, 40 \mathrm{~A}, 75 \mathrm{~A}$ and $150 \mathrm{~A} \mathrm{GeV} / \mathrm{c}$, in the framework of Angantyr model [17]. Angantyr adds a framework wherein AA-collisions can be constructed as a superposition of binary nucleon-nucleon collisions as implemented in PYTHIA8 event generator [18]. For this study we have used the PYTHIA/Angantyr 8.303 (released in September 2020), which has the latest version of hadron rescattering ( $\mathrm{HadSc}$ ) model [19]. The main ingredients of the model consist of four components: initial conditions, parton level configurations, hadronisation and primary hadronic interactions. The initial conditions include the spatial and momentum distributions of partons and excited strings. The generation of partonic level 
activities, involve initial and final state radiations, multiple parton interactions (MPI) and beam remnants. At the end of this step, a partonic structure has been created. The hadronisation of this parton configuration is obtained when partons stop interacting, and the resulting strings are converted to hadrons using Lund string fragmentation scheme [20-22]. Beyond that, Angantyr offers a platform on top of which various collective non-QGP effects can be added. One example is the thermal model $[23,24]$, whereby closely backing of nearby strings leads to an increased effective temperature and thereby change both particle composition and the transverse momentum spectra.

Both the standard fragmentation [20] and thermal models [23] produce a region of closely overlapping hadrons, that then can collide with each other as the system expands. In this work, primary hadron rescattering (HadRSc) [23] will also be employed especially in light(er)-ion beam collisions, where visible physics signals are expected.

The manuscript is organized as follows: in Sect. 2, we define the basic ingredients of the Angantyr model. In Sect. 3 the effects of standard fragmentation and thermal models with/without HadRSc are tested and confronted to $\pi^{-}$ rapidity and transverse mass spectra in inelastic pp and most central ${ }^{7} \mathrm{Be}+{ }^{9} \mathrm{Be}$ and ${ }^{40} \mathrm{Ar}+{ }^{45} \mathrm{Sc}$ collisions at CERN SPS energies $[9,10,12]$. Finally, in Sect. 4 we present our conclusions.

\section{Description of the model}

Angantyr [17] is a Monte Carlo Model for heavy-ion collisions included in PYTHIA8 [18]. It extrapolates protonproton (pp) dynamics, described by PYTHIA8 event generator [18], to proton-nucleus (pA) and nucleus-nucleus (AA) collisions. Angantyr does not include flow or jet quenching. As such, it is a good baseline for collisions in the absence of a QGP.

The Angantyr part of PYTHIA8 modeling is responsible for setting up the AA collision geometry, and selecting the number of NN-(sub)collisions. The nucleon locations inside a nucleus are sampled according to a Wood-Saxon distribution in the GLISSANDO (Glauber initital state simulation and more) parameterizations [25]. The generalization to AA-collisions is inspired by the notion of wounded (participating) nucleons [26]. The number of wounded nucleons is calculated from an advanced Glauber model, including Gribov corrections of diffraction excitation of interacting nucleons [27]. The model is formulated for two interaction types: soft nucleon-nucleon (NN) collisions treated as in FRITIOF [20-22], and multiple hard partonic subcollisions treated as in PYTHIA8 [18].

Fluctuations in nuclear radii are taken into account, which can reproduce fluctuations in $\mathrm{NN}$-cross section and allows for an extension to pA and AA collisions. Each individual NN subcollisions is characterised as diffractive or non-diffractive collisions. In particular, the fluctuation color model puts the distinction between primary and secondary absorptive nucleons. The procedure starts by looking at all pairs of interacting projectile/target nucleons and ordered them in a list in increasing NN-impact parameter. Depending on impact parameter, one determines a set of primary absorptive collisions and a set of secondary ones (where one of the participating nucleons has already been interacted in a primary sub-event). For each of the primary ones, an inelastic nondiffractive minimum bias events in PYTHIA8 are generated. The secondary absorptive subcollisions are generated by PYTHIA8 with a special diffraction like treatment. As described in [17], for high-mass diffraction $\left(M_{X}>10 \mathrm{GeV}\right)$ PYTHIA treats the single diffraction (SD) excitation as a nondiffraction (ND) interaction between the target nucleon and a Pomeron emitted from the projectile using the full multiparton interaction (MPI) machinery as if the Pomeron was a hadronic object with parton densities.

For low-mass diffraction $\left(M_{X} \simeq 10 \mathrm{GeV}\right)$, the excited system is modeled as a simple longitudinal stretched string and simulated using the assumptions of FRITIOF model [2022]. It is assumed that an interacting nucleon suffers a longitudinal momentum exchange with a distribution $d P^{ \pm} / P^{ \pm}$, where $P^{+}$and $P^{-}$are the light cone momenta in the direction of the projectile and target, respectively. The distribution of diffractive masses used in the generation of soft $N N$ collisions is thus given by $\sim d M_{X}^{2} / M_{X}^{2}$.

The fragmentation stage of the model now takes over and convert the colored partonic state into a set of colorless hadrons. Quarks become string endpoints, while gluons become transverse kinks connecting two string pieces. The Lund string model [28] describes the fragmentation of such string systems into full hadrons. The corner stone of the Lund model [28] is the observation that the QCD potential between a quark and antiquark form a high tension string (flux tubes), with tension $k_{0}=1 \mathrm{GeV} / \mathrm{fm}$. As the quark $q$ and antiquark $(\bar{q})$ move apart, the potential energy stored in the string increases, and the string may break by the production of a new "vertex" pair $q^{\prime}-\bar{q}^{\prime}$, such that a meson $\left(q \bar{q}^{\prime}\right)$ is formed and $q^{\prime}$ is left. This $q^{\prime}$ may pair off with a $\bar{q}^{\prime \prime}$ and so on.

The break-up probability of a $q \bar{q}$ pair is given by the quarklevel Gaussian suppression of the form [28]

$\exp \left(-\pi m_{\perp q}^{2} / k\right)=\exp \left(-\pi m_{q}^{2} / k\right) \exp \left(-\pi p_{\perp q}^{2} / k\right)$,

with transverse width $\sigma_{q^{\prime} q^{\prime}}^{2}=k / \pi$, where $k$ is the string tension. The transverse momentum of a hadron is given by a vertex diquark $p_{T}$ kick involved in string breaking and characterized by a Gaussian width $\sigma_{q^{\prime} q^{\prime}}$, with $\sigma_{q^{\prime} q^{\prime}}=0.335 \mathrm{GeV} / \mathrm{c}$ and a small $(1 \%)$ tail of breaks involving higher $p_{T}$ values 
[4]. Baryon-antibaryon $(B \bar{B})$ pairs are also introduced in the Lund popcorn mechanism by allowing diquark-antidiquark breakups of the string, i.e., the quarks-antiquarks in the $B \bar{B}$ pairs are popping out one pair at a time from the color force field. In the case the fluctuations are large on the scale of meson masses it is possible that one or more mesons are produced between the $B \bar{B}$ pairs. In the implementation we adopt the Monash 2013 tunes that govern quark-level suppression of the light meson and baryon production [30].

In the PYTHIA thermal model [23], the quark-level Gaussian suppression is replaced by an hadron-level exponential one of the form

$\exp \left(-m_{\perp \text { had }} / T\right)$

with $m_{\perp \text { had }}=\sqrt{m_{\text {had }}^{2}+p_{\perp \text { had }}^{2}}$. The width $T$ is associated with the temperature in the context of thermodynamical model. Also, $T \sim \sqrt{k / \pi}=\sigma$ could be viewed as the string energy per unit length. Note that, the Lund longitudinal string fragmentation function remains unchanged. In the thermal model, only light flavor ( $u, d$ and $s$ ) mesons and baryon multiplets are taken into account. Popcorn baryon production mechanisms are also not implemented in the model.

The longitudinal momentum $\left(p_{z}\right)$ carried by a hadron formed in the string breaking process remains unchanged in both the Lund and thermal models and is governed by the symmetric fragmentation function [29]

$f(z) \propto \frac{1}{z}(1-z)^{a} \exp \left(-\frac{b m_{\perp}^{2}}{z}\right)$,

where $z$ is the light-cone longitudinal momentum fraction of the newly produced hadron with transverse mass $m_{\perp}$, expressed as a fraction of the light-cone energy of the (anti)quark endpoint $i$ of the fragmenting string. The remaining (light-cone) energy fraction $(1-z)$ goes to the new string system, from which another hadron can be split off until all the energy is used up. We employ the PYTHIA 8.303 default values of $a=0.68$ and $b=0.98 \mathrm{GeV}^{-2}$.

At the earliest times after hadronization, PYTHIA8 includes the possibility for hadrons to rescatter on the way out [19]. This is a consequence of the PYTHIA model for MPI and the string model of particle production. The model is applied the primary low $p_{\perp}$ hadrons which are produced directly from the string fragmentation, before secondary decays are considered. Each primary hadron has been assigned a production vertex $x_{0}=\left(t_{0}, x_{0}\right)$ and a four momentum $p=(E, \mathbf{p})$. It is assumed that all primary hadrons move along straight line trajectories $x(t)=x_{0}+\left(t-t_{0}\right) \mathbf{p} / E$. The probability of an interaction of two particle species in impact parameter space $\mathbf{b}$ is given by [19]

$P=P_{0} \exp \left(-b^{2} / b_{0}^{2}\right)$, where $P_{0}=0.75$ is the opacity and $b_{0}=\sqrt{\frac{\sigma_{\text {tot }}}{P_{0} \pi}}$ is the characteristic length scale. The total cross section $\sigma_{\text {tot }}$ depends on the species and quantum number of the scattered particles. The cross section for different collision processes include: (i) long lived hadrons such as $\pi, K, \eta, \eta^{\prime}, p, n, \Lambda, \Sigma, \Xi$ and $\Omega$, and (ii) short lived hadrons such as $\rho, K^{*}, \omega, \phi, \Delta$, $\Sigma^{*}$ and $\Xi^{*}$. Each hadron pair of an event is first checked to see if it fulfills Eq. 4 and, if it does, the interaction time for that pair is recorded in a time ordered list. Then the scattering that is first in order is simulated unless the particles involved have already interacted. This produces new hadrons that checked for rescatterings against the other particles, and any such interactions are inserted into the time ordered list. This process is repeated until there are no more potential interactions.

Calculations in Angantyr consists of Glauber-Gribov (GG) color fluctuations to generate the number of subcollisions, PYTHIA8 multiple parton interactions (MPI), beam remnants and string fragmentations models. The results of these models depend on a variety of parameters, whose best settings can be obtained from tuning pp-collisions with respect to measured data. The default Monash 2013 tunes [30] of PYTHIA8 are known to describe a large set of Large Electron-Positron Collider (LEP), Tevatron and The Large Hadron Collider (LHC) data (in what follows refereed to as Tune A, see Table 1 of Appendix). For the MPI settings, it assumes $p_{\perp 0}=2.28 \mathrm{GeV} / \mathrm{c}$ (MultipartonInteractions:pT0Ref $=2.28)$ at the reference energy $1.8 \mathrm{TeV}$ (MultipartonInteractions:ecmRef $=1800$ ), with an energy rescaling proportional to $s^{\epsilon}(\epsilon=$ MultipartonInteractions:ecmPow $=0.215)$. The impact parameter is assumed to be a convolution of an exponential distribution of the two incoming proton beams (MultipartonInteractions:bProfile $=3$ ). As for the beam remnants, the transverse momentum of primordial initiators of hard subcollisions are selected according to a Gaussian distribution. The width of this distribution depends on the hard scale $(Q)$ of the central process, the soft $\left(\sigma_{\text {soft }}\right)$ and hard $\left(\sigma_{\text {hard }}\right)$ interaction limits as well as the half-way point $\left(Q_{1 / 2}\right)$ between the hard and soft processes. The values of these parameters are taken as $\sigma_{\text {soft }}=$ BeamRemnants:primordialKTsoft $=0.9, \sigma_{\text {hard }}=$ BeamRemnants:primordialKThard $=1.8$, and $Q_{1 / 2}=$ BeamRemnants:halfScaleForKT $=1.5$. The values of Lund string fragmentation function parameters $\left(\alpha_{i(j)}\right.$ and $\left.b\right)$ are taken to be $\alpha_{i(j)}=$ StringZ: aLund $=0.68$ and $b=$ StringZ:bLund $=0.98$. The diquark $\left(\gamma_{q q}\right)$ and strangeness $\left(\gamma_{s}\right)$ suppression factors are given by $\gamma_{q q}=$ StringFlav:probQQtoQ $=0.081$ and $\gamma_{s}=$ StringFlav:probStoUD $=0.217$. Finally, the values of hadron rescattering ( $\mathrm{HadSc}$ ) model is given by $P_{d s}^{\max }=$ HadronScatter:maxProbDS $=0.5, P_{s S}^{\min }=$ HadronScatter $: \operatorname{minProbSS}=$ 0.5 and $P_{s s}^{\max }=$ HadronScatter:maxProbSS $=1.0$. 
The PYTHIA Gaussian and thermodynamical models are simulated by modifying PYTHIA 8.303 with Monash tune for pp-collisions at the lowest SPS beam momentum of 20 $\mathrm{GeV} / \mathrm{c}$. The thermal model will be used as the prototypical model of collective effects. In Sect. 3, we will show how the Gaussian and thermal settings are tuned at low SPS energies and limit ourselves to general bulk properties such as rapidity and transverse momentum distributions. The settings of Table 1 (without HadSc model) introduces first changes with respect to the default Monash 2013 tunes to obtain the results for pp-collisions. The whole settings of Table 1 denote the PYTHIA Gaussian and thermal calculations with primary hadron rescatterings. We want to emphasize at the beginning that these settings are tuned to pp data, and no tuning is done to ${ }^{7} \mathrm{Be}+{ }^{9} \mathrm{Be}$ and ${ }^{40} \mathrm{Ar}+{ }^{45} \mathrm{Sc}$ data. The Angantyr results of variations to the hadronization model can thus be regarded as real predictions depending on the chosen parameter set.

\section{Results and discussion}

In this section we present the predictions of the PYTHIA Angantyr code [17] compared with the data from NA61/SHINE measurements of the rapidity distributions and transverse momentum spectra of pions in proton-proton and central ${ }^{7} \mathrm{Be}+{ }^{9} \mathrm{Be}$ and ${ }^{40} \mathrm{Ar}+{ }^{45} \mathrm{Sc}$ collisions at incident projectile momentum of 19, 30, 40, 75 and $150 \mathrm{~A} \mathrm{GeV/c}$ (which corresponds to $\sqrt{s_{\mathrm{NN}}}=6.1,7.6,8.8,11.9$ and $16.8 \mathrm{GeV}$, respectively) [9-12].

Data on pion production are crucial for constraining basic properties of Monte Carlo event generators. Before studying pion production in central light(er)-ion beams at SPS energies, it is necessary to verify that PYTHIA Angantyr for ppcollisions works well with the corresponding experimental data, which are taken with the same detector and same acceptance. Figures 1 and 2 compare PYTHIA 8.303 results, based on Monash 2013 tunes [30], with the NA61/SHINE data for the inclusive $\pi^{-}$meson spectra in inelastic pp-collisions at beam momenta of 20,40,80 and $158 \mathrm{GeV} / \mathrm{c}$ [9]. As one can see, the $\pi^{-}$particle $p_{\perp}$ spectra at mid-rapidity $(0<y<0.2)$ are not correctly modeled at large $p_{\perp}$ scales. The issue shows up in rapidity $(d N / d y)$ distributions, where it is not possible to obtain a good description for data with increasing collision energy.

A mismatch in the $\pi^{-}$meson spectra is easily compensated by tunning the MPI modelling, see Figs. 1 and 2. The $p_{\perp \text { min }}^{2}$ scale of the regularization factor $p_{T}^{4} /\left(p_{\perp \text { min }}^{2}+p_{T}^{2}\right)^{2}$ is used to tame the dampening of the MPI cross section $\left(\sigma_{\text {int }}\right)$ at $p_{\mathrm{T}} \rightarrow 0$. In the default PYTHIA 8.303, $p_{\perp \min }^{2}$ is given by [18]

$p_{\perp \text { min }}^{2}(s)=p_{\perp 0}^{2}\left(\frac{s}{s_{\mathrm{ref}}}\right)^{\epsilon}$,

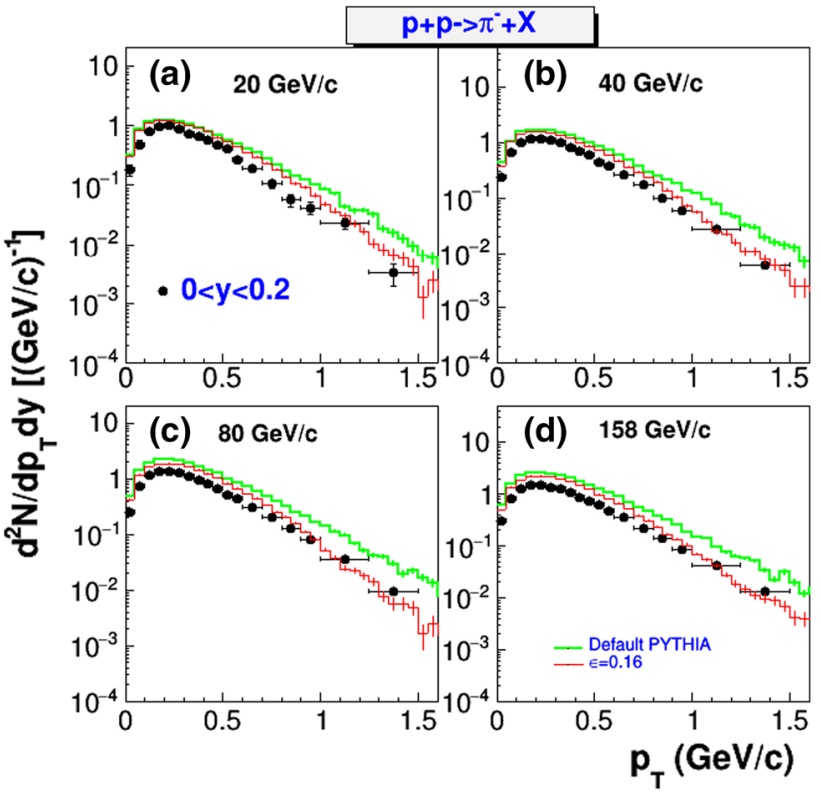

Fig. 1 Transverse momentum specta of $\pi^{-}$in inelastic pp collisions at beam momentum of $20,40,80$ and $158 \mathrm{GeV} / \mathrm{c}$. The thick and thin lines denote the default PYTHIA8.303 and Gaussian $p_{\mathrm{T}}$ settings of Table 1, respectively. The closed points represent NA61/SHINE [9] data

with $p_{\perp 0}=2.28 \mathrm{GeV} / \mathrm{c}$ and some power $\epsilon$ that sets the scaling away from $s=s_{\text {ref }}=E_{C M}^{2}$. The default value for the scaling power in PYTHIA 8.303 was $\epsilon=0.215$ for the $E_{C M}=7000 \mathrm{GeV}$ reference energy. The settings that are varied in our tune are the reference energy, $s_{\text {ref }}$, and the energy dependence scaling $(\epsilon)$. We change the reference energy to be $E_{C M}=10 \mathrm{GeV}$ to control the power law extrapolation as much as possible at low center of mass energies. As one can see in Figs. 1 and 2, a mismatch in the energy dependence of $\pi^{-}$spectra can be compensated by the choice of $\epsilon=0.16$, which resulted in too little activity at CERN SPS energies. The value $\epsilon=0.16$ is motivated by relating it to the scaling of the energy dependence of the total $p p$ cross section $\left(\sigma_{\text {tot }}\right)$ via Regge phenomenology [31], which grows like $\propto E_{\mathrm{cm}}^{0.16}$. It should be pointed out here that the PYTHIA 8.303 total cross section is obtained from a Donnachie-Landshoff fit and is not affected by the choice of $\epsilon$ [32].

In Fig. 3 we show the transverse mass spectra of $\pi^{-}$ mesons produced at mid-rapidity $(0<y<2)$ in inelastic pp-interactions at beam momenta of 20, 31, 40 and 158 $\mathrm{GeV} / \mathrm{c}$. The symbols correspond to NA61/SHINE data and the small-dashed lines to PYTHIA with Gaussian $p_{\perp}$ suppression with $<p_{\perp \mathrm{q}}^{2}>=(0.31 \mathrm{GeV})^{2}$. One observes that PYTHIA with Gaussian model describes well the transverse mass spectra in the range $0.2<m_{\mathrm{T}}-m_{\pi}<0.7 \mathrm{GeV}$, where no strong contributions from resonance decays and radial flow are expected. However, a specific pattern of deviations of the $m_{\mathrm{T}}-m_{\pi}$ spectra emerges between the model and data, where the low and high $m_{\mathrm{T}}-m_{\pi}$ pion yield go respec- 


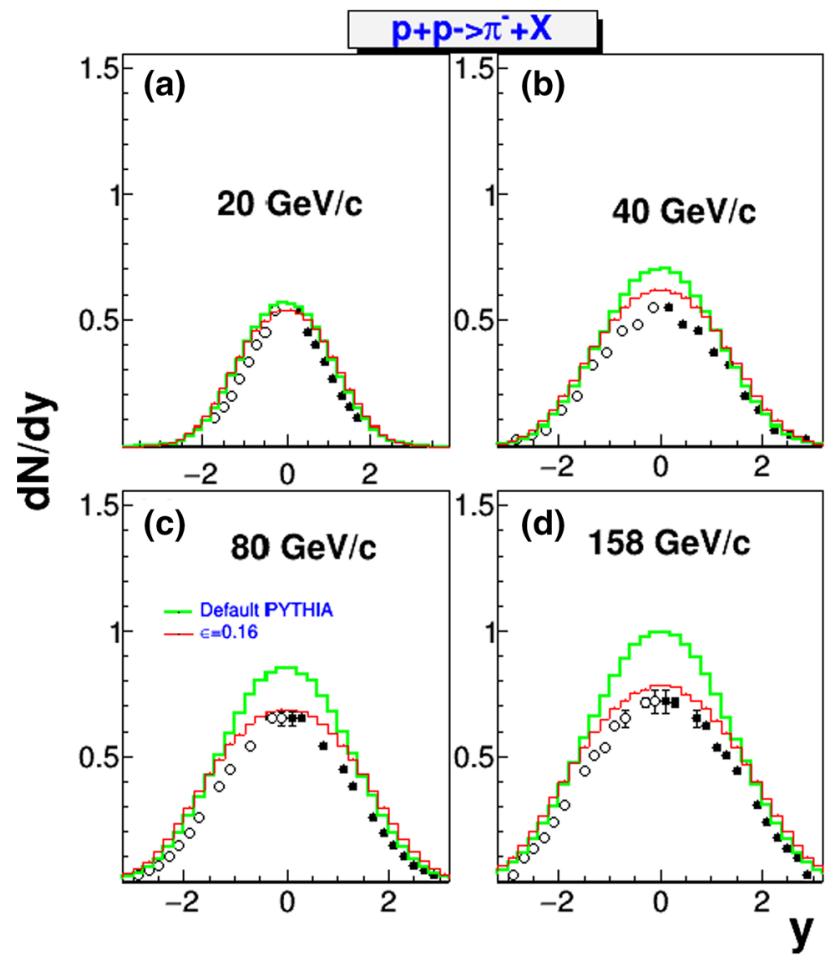

Fig. 2 Same as Fig. 1, but for the rapidity distributions. The open points symmetrize NA61/SHINE data [9]

tively above and below the experimental data at the SPS energies. This implies that the well tested PYTHIA hadronization model fail to reproduce the basic bulk observables like the $m_{\mathrm{T}}$ spectra of $\pi^{-}$in pp collisions.

To explore whether thermal hadronization effects could contribute to the resolution of the effects that we are attempting to explain in pp-collisions, we include in Fig. 3 (see solid lines) thermal PYTHIA calculations. Comparing the $m_{\mathrm{T}}-m_{\pi}$ spectra for the Gaussian $p_{\perp}$, we notice that the effects of thermalization leads to an enhancement of the spectra at $m_{\mathrm{T}}-m_{\pi}>0.7 \mathrm{GeV}$, in accord with the experimental data. This implies that the exponential hadron-level $m_{T}$ suppression effects are able to push up the predictions for higher $m_{T}$ values, compared to the quark-level Gaussian one. On the other hand, both calculations overshoot the distributions at smaller $m_{\mathrm{T}}$ values, especially as the collision energy increases. The observed discrepancy at low $m_{\mathrm{T}}$ is largely a result of resonance decay contributions. It was shown in Ref. [33] that hydrodynamical model including transverse flow and resonances having two- or three-body decays such as $\rho$, $K_{S}^{0}, K^{*}, \Delta, \Sigma+\Lambda, \eta, w$ and $\eta^{\prime}$ account for the transverse mass spectra of pions at low $m_{\mathrm{T}}$ values for central $\mathrm{Pb}+\mathrm{Pb}$ collisions at $158 \mathrm{AGeV}$.

The effects of change of the temperature $(T)$ of the exponential hadron level suppression, Eq. 2, are illustrated in Figs. 3 and 4. As seen from Fig. 4, the pion rapidity distribution is sensitive to the temperature: the experimental data are in best

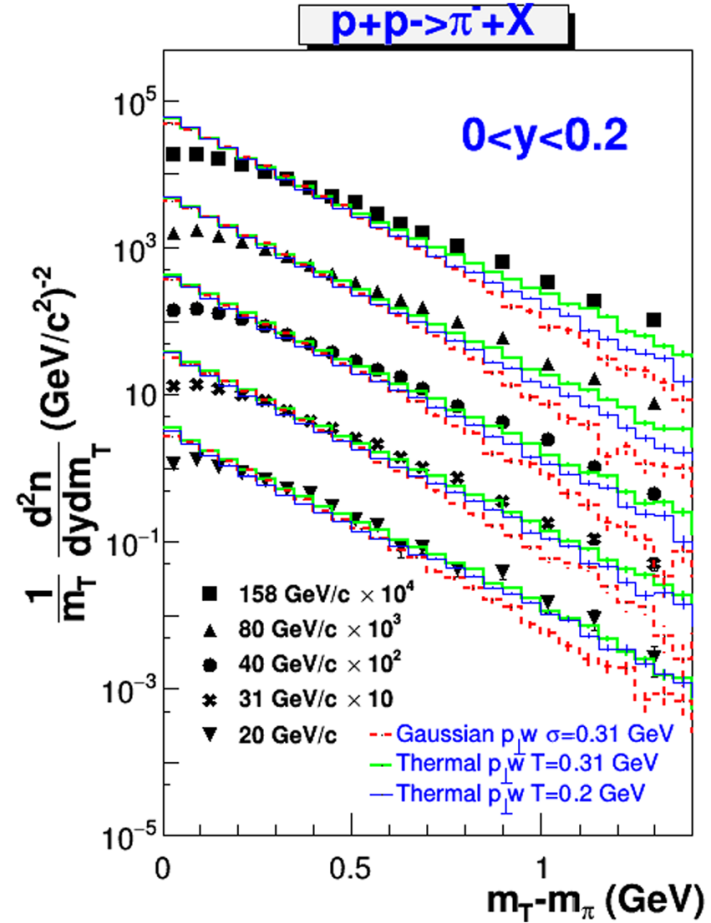

Fig. 3 The transverse mass spectra of $\pi^{-}$in inelastic pp-collisions at mid-rapidity $(0<y<0.2)$. The small-dashed and solid lines denote the Gaussian and thermal models with adjusted width $\sigma$ and temperature $(T)$, respectively. The closed points represent NA61/SHINE [9] data. For better readability, both the calculations and data points were scaled

agreement with thermodynamical model using lower temperature value of $T=0.2 \mathrm{GeV}$ at mid-rapidity, but clearly broadens the distributions as the collision energy increases. On the other hand, a higher temperature increases the slope of the spectra at large $m_{\mathrm{T}}$ as compared to a smaller one, see Fig. 3, in accordance with experimental data. The adjusted parameter values of pp-collisions can be found in Appendix A.

After obtaining suitable sets of parameters in the PYTHIA Angantyr model for pp-collisions, we have calculated the rapidity and transverse momentum and mass distributions of pions in central ${ }^{7} \mathrm{Be}+{ }^{9} \mathrm{Be}$ and ${ }^{40} \mathrm{Ar}+{ }^{45} \mathrm{Sc}$ collisions at beam momenta of 13A, 19A, 30A, 40A, 75A and 150A GeV/c. The ${ }^{7} \mathrm{Be}+{ }^{9} \mathrm{Be}$ collisions provide an unique possibility to explore such lowest mass isospin symmetric nuclear system. It is also noted in Ref. $[23,24]$ that the yield ratio of strange hadrons to pions in these collisions gives close values to those in central $\mathrm{Pb}+\mathrm{Pb}$ collisions and significantly higher than in pp-interactions. The NA61/SHINE results on $\mathrm{Ar}+\mathrm{Sc}$ interactions are also interesting since an increased effective number of degrees of freedom is expected at the highest SPS energies. In particular, the pion mean multiplicity is found equal that for inelastic pp-reactions at low SPS energies whereas it is closer to that for $\mathrm{Pb}+\mathrm{Pb}$ collisions at high SPS energies. We use for the centrality selection events the range of impact 
Fig. 5 Rapidity distributions of protons in the $20 \%$ most central ${ }^{7} \mathrm{Be}+{ }^{9} \mathrm{Be}$ collisions at different SPS energies. Predictions of the PYTHIA 8.303/Angantyr calculations using Gaussian and thermal models are shown in thin and short-dashed lines, respectively. The data have been symmetrized. The closed points represent NA61/SHINE [10] data and open points are symmetrized

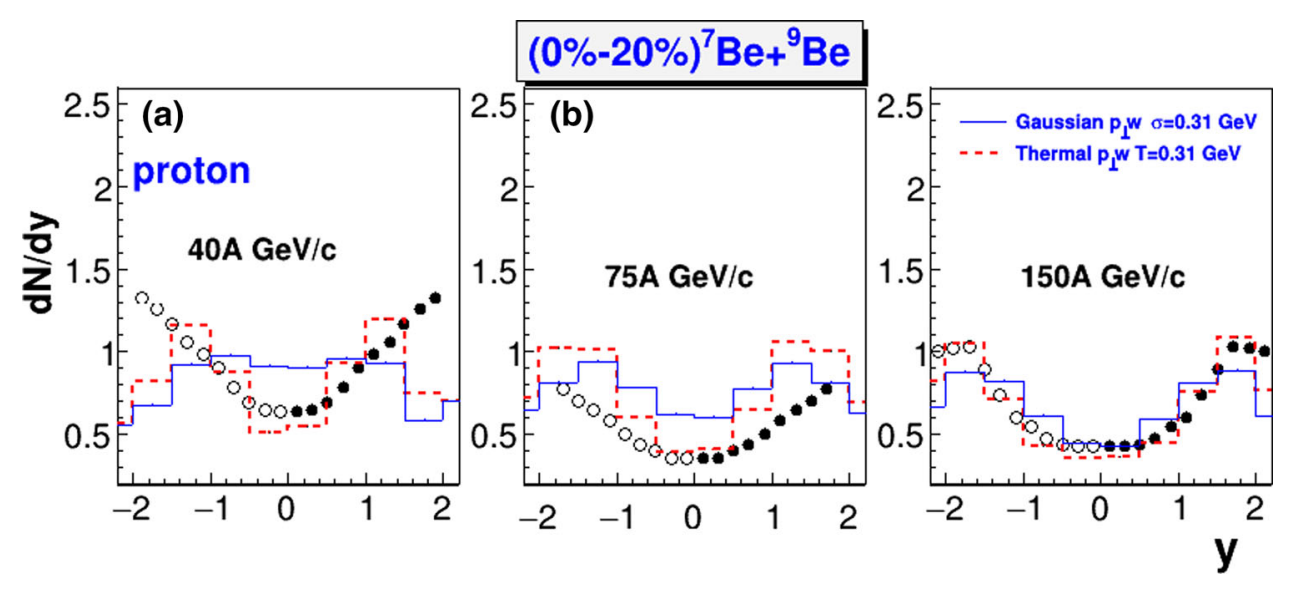

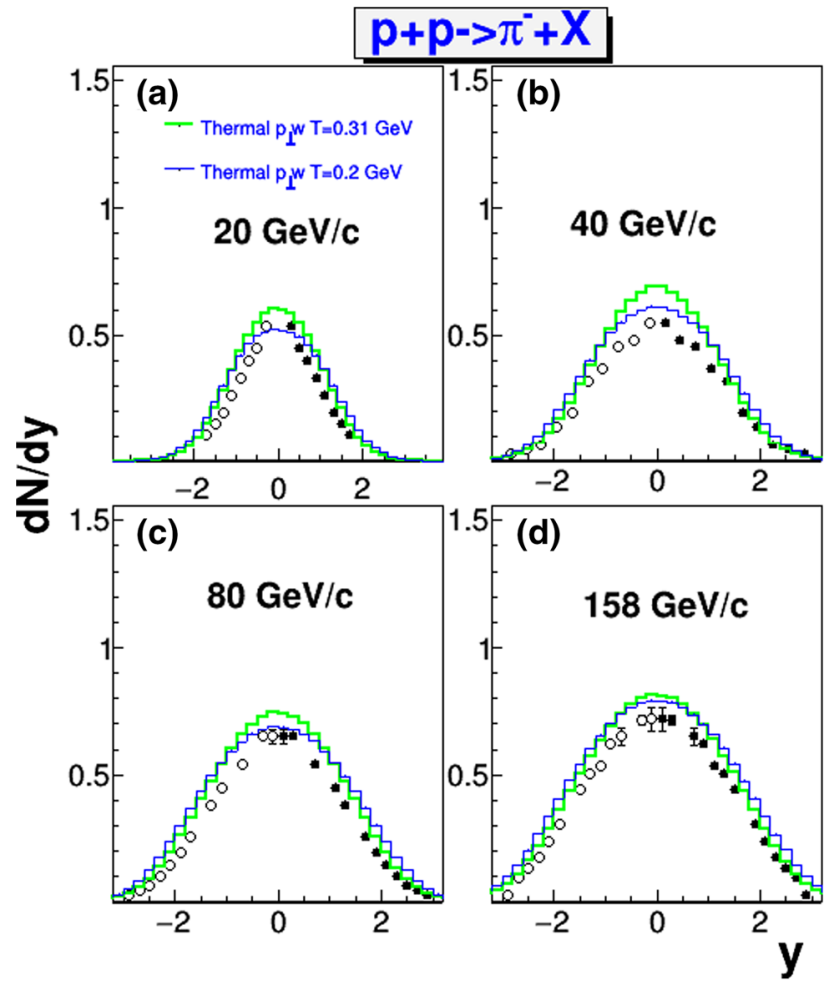

Fig. 4 Rapidity distributions of $\pi^{-}$in pp collisions in comparison to PYTHIA 8.303 calculations with different temperatures (T). The closed points represent NA61/SHINE [10] data and open points are symmetrized

parameters provide by NA61/SHINE [12] and not the one extracted from PYTHIA Angantyr. It should be pointed out that a harmonic oscillator shell model distribution, to simulate nucleon positions for light nuclei with mass number $3 \leq A \leq 16$, is not implemented in PYTHIA8, and here we use the Wood-Saxon distribution with GLISSANDO parameterizations [25].

We start our comparison by displaying in Fig. 5 the proton rapidity distributions in $0-20 \%$ central ${ }^{7} \mathrm{Be}+{ }^{9} \mathrm{Be}$ collisions at $\sqrt{s_{\mathrm{NN}}}=8.8,11.9$ and $16.8 \mathrm{GeV}$, calculated in Angantyr using both the Lund-Gaussian and thermal models. It is seen that the calculations using the Gaussian model (thin lines) describes well the measured $d N / d y$ of protons at midrapidity for $\sqrt{s_{\mathrm{NN}}}=16.8 \mathrm{GeV}$. However, protons obtain too much stopping as the collision energy decreases. On the other hand, the thermal model calculations (thin-dashed lines) provide a correct stopping at different CERN SPS energies. This illustrates the limitation of the applicability of partonlevel Gaussian suppression for lighter ion beams at lower SPS energies. The agreement of Angantyr calculations using exponential suppression is important for the interpretation of results of pions, since their abundances are sensitive to the energy loss of the initial colliding nucleons, i.e., to the fraction of their kinetic energy which will be converted into mass production.

In Figs. 6 and 7 the $y$ and $p_{\mathrm{T}}$ distributions of pions for $20 \%$ central ${ }^{7} \mathrm{Be}+{ }^{9} \mathrm{Be}$ collisions at different SPS energies are presented for the Gaussian and thermodynamical models along with the corresponding experimental data from NA61/SHINE collaboration [10]. As seen from Fig. 6, the thermal model leads to an enhancement of $\pi^{-}$distributions at mid-rapidity as compared to the Gaussian model, in agreement with the data. Figure 7 shows that the difference between the Gaussian and thermodynamical models becomes more pronounced at smaller $p_{\mathrm{T}}$ values. We also find that the spectra of $\pi^{ \pm}$are well described in the Angantyr approach for both models at different SPS energies.

Other models have also been used to study $p_{\mathrm{T}}$ spectra at midrapidity and rapidity spectra of $\pi^{ \pm}$and protons in the $20 \%$ most central ${ }^{7} \mathrm{Be}+{ }^{9} \mathrm{Be}$ collisions at SPS energies. The UrQMD (version 3.4) model [5] (which generate hadron cascade based on elementary cross sections for resonance production which either decay (at low energies) or converted into strings which fragment into hadrons (at high energies) accompanied by final hadron scattering) has been compared to the NA61/SHINE data. It is demonstrated in reference [10] that the UrQMD overestimates the $p_{\mathrm{T}}$ spectra of protons and $\pi^{-}$in the $20 \%$ most central ${ }^{7} \mathrm{Be}+{ }^{9} \mathrm{Be}$ collisions at different SPS energies. On the other hand, the EPOS calculations [16], which includes collective expansion of a thermal- 

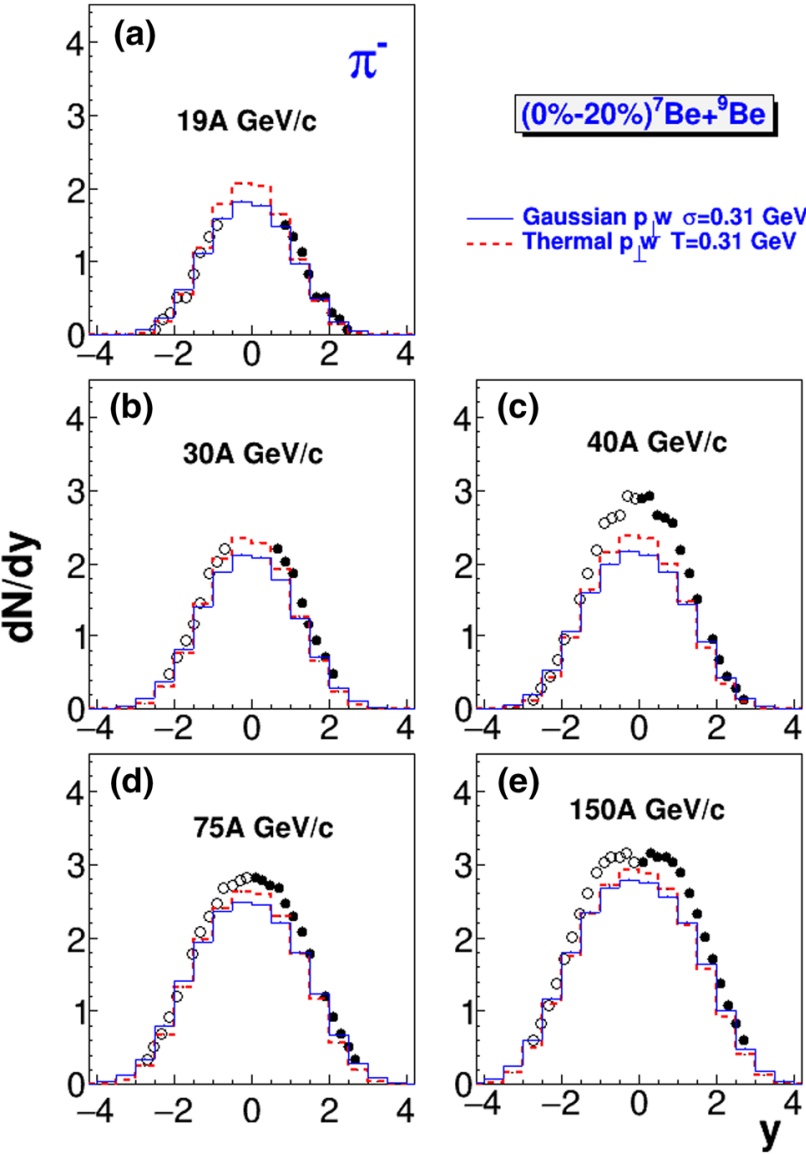

Fig. 6 Rapidity distributions of negative pions in the $20 \%$ most central ${ }^{7} \mathrm{Be}+{ }^{9} \mathrm{Be}$ collisions. Predictions of the PYTHIA 8.303/Angantyr calculations using Gaussian and thermal models are shown in thin and short-dashed lines, respectively. The data have been symmetrized. The closed points represent NA61/SHINE [10] data and open points are symmetrized

ized medium, the two-off remnants and elementary scattering represented by a parton ladder, reproduce rather well the measured proton $d N / d y$ and $p_{\mathrm{T}}$ spectra, while they overestimate the $\pi^{ \pm}$and $K^{ \pm}$rapidity spectra at the highest SPS energy. The AMPT (version 1.26) [7], the PHSD (version 4.0) [34] also fail in describing the rapidity distribution of the NA61/SHINE data.

It should also be noted that the Simulating Many Accelerated Strongly-interacting Hadrons (SMASH) model [35], based on a relativistic Boltzmann equations with binary interactions, has been compared to the NA61/SHINE measurements of the studied interactions. It is demonstrated in reference [10] that SMASH version 1.6, with parameters tuned to match measurements in pp-collisions, describes well the $p_{\mathrm{T}}$ spectra of the identified hadron. As for the rapidity distributions, SMASH, however, overpredicts $\pi^{-}$yield by a factor of $\sim 25 \%$ at midrapidity.

We step up to heavier systems and confront expectations from Angantyr with the available experimental data at SPS

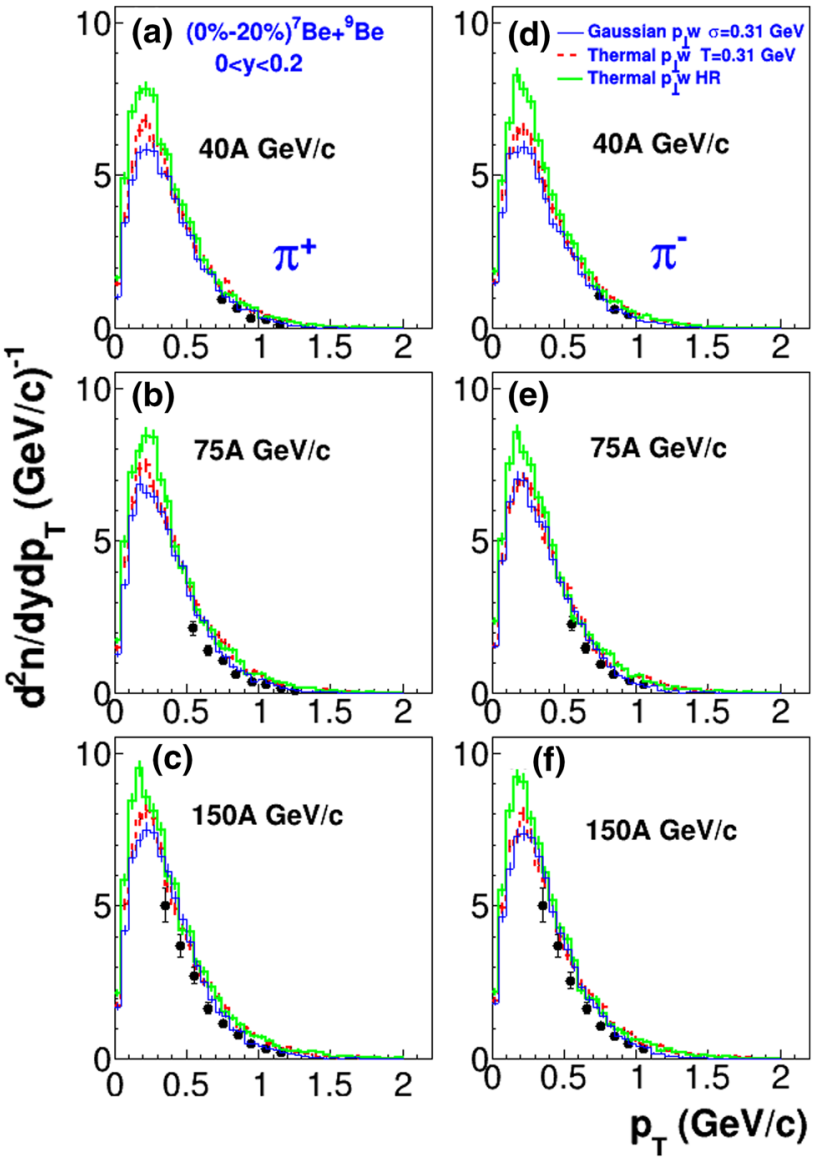

Fig. 7 Same as Fig. 6 but for the transverse momentum spectra of $\pi^{ \pm}$ at mid-rapidity $(0<y<0.2)$

energies. In Fig. 8 the Angantyr results with Gaussian and thermal models for the rapidity distributions of $\pi^{-}$in $5 \%$ central ${ }^{40} \mathrm{Ar}+{ }^{45} \mathrm{Sc}$ collisions at the whole SPS energies are compared to the corresponding data from NA61/SHINE [12]. It is seen that the thermodynamic model describes the measured $d N / d y$ of $\pi^{-}$very well at low SPS energies ( $\leq$

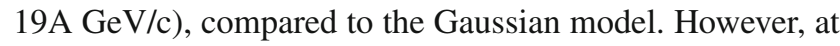
higher SPS energies both models undershoot the distributions at mid-rapidity, especially as the collision energy increases.

Finally, in Figs. 9 and 10 we investigate within the context of string hadronization model, some pion enhancement variables, considering primary hadron rescattering $(\mathrm{HadSc})$. In this respect Angantyr code provides an unique possibility to explore the possibility that fragmenting strings are closely packed, which implies the initial formation of a dense hadronic gas, wherein rescattering may lead to collective like effects. Compared to the $\pi^{-} d N / d y$ calculations using the Gaussian and thermal models, we notice that the $\pi^{-}$distributions get broader with HadSc as shown in Fig. 9, i.e., we get more pions at midrapidity. The thermal/HadSc model is shown to provide a better description of the measured $\pi^{-}$ $d N / d y$ at mid-rapidity for beam energies of $30 \mathrm{~A}$ and $40 \mathrm{~A}$ 
$5 \%$ Ar + Sc $->\pi^{-}+\mathrm{X}$
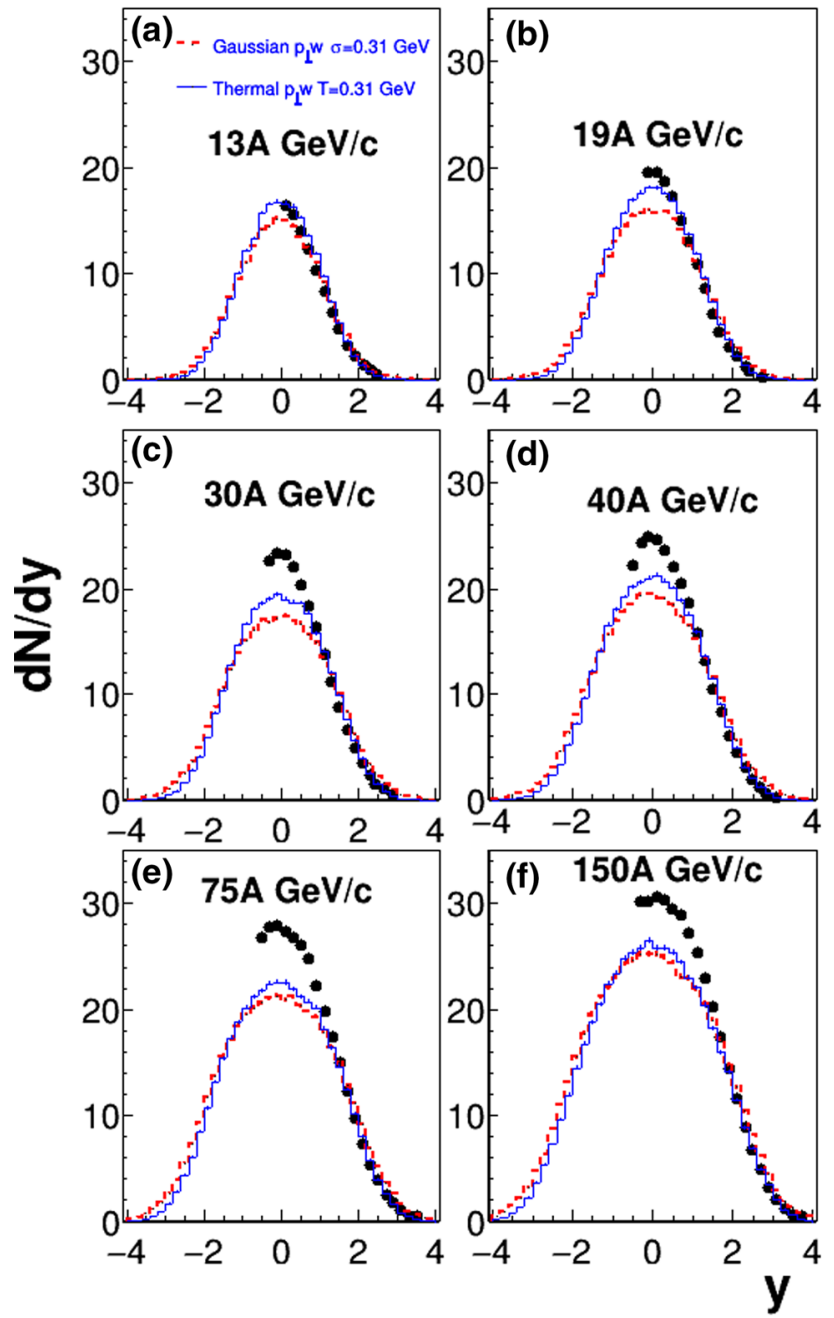

Fig. 8 Rapidity distributions of $\pi^{-}$in the $5 \%$ most central ${ }^{40} \mathrm{Ar}+$ ${ }^{45} \mathrm{Sc}$ collisions at beam momenta of 13A, 19A, 30A, 40A, 75A and $150 \mathrm{~A} \mathrm{GeV} / \mathrm{c}$. The solid and small-dashed lines denote the Gaussian and thermal models, respectively, tuned to pp-parameters of Table 1. The closed points represent NA61/SHINE data [12]

$\mathrm{GeV} / \mathrm{c}$, compared to the Gaussian/HadSc model. This indicates that the rapid changes of hadron production properties observed in central AA-collisions at about $30 \mathrm{~A} \mathrm{GeV/c}$ are sensitive to thermal/HadSc hadronization model. It is also shown in Fig. 9 that the Gaussian/HadSc model produces a broad $\pi^{-}$distributions at $y>1.5$ as the beam energy increases. On the other hand, the thermal/HadSc model reduces the $\pi^{-}$yield in this region and more closely reproduces the experimental data.

In Fig. 10 we examine the $m_{\mathrm{T}}$ spectra of $\pi^{-}$particles at mid-rapidity $(0<y<0.2)$ rapidity for all six beam momenta. Similar to the previous observables, the thermal/HadRSc model improves the description of the measured $m_{\mathrm{T}}$ spectra, compared to the Gaussian/HadRSc, as

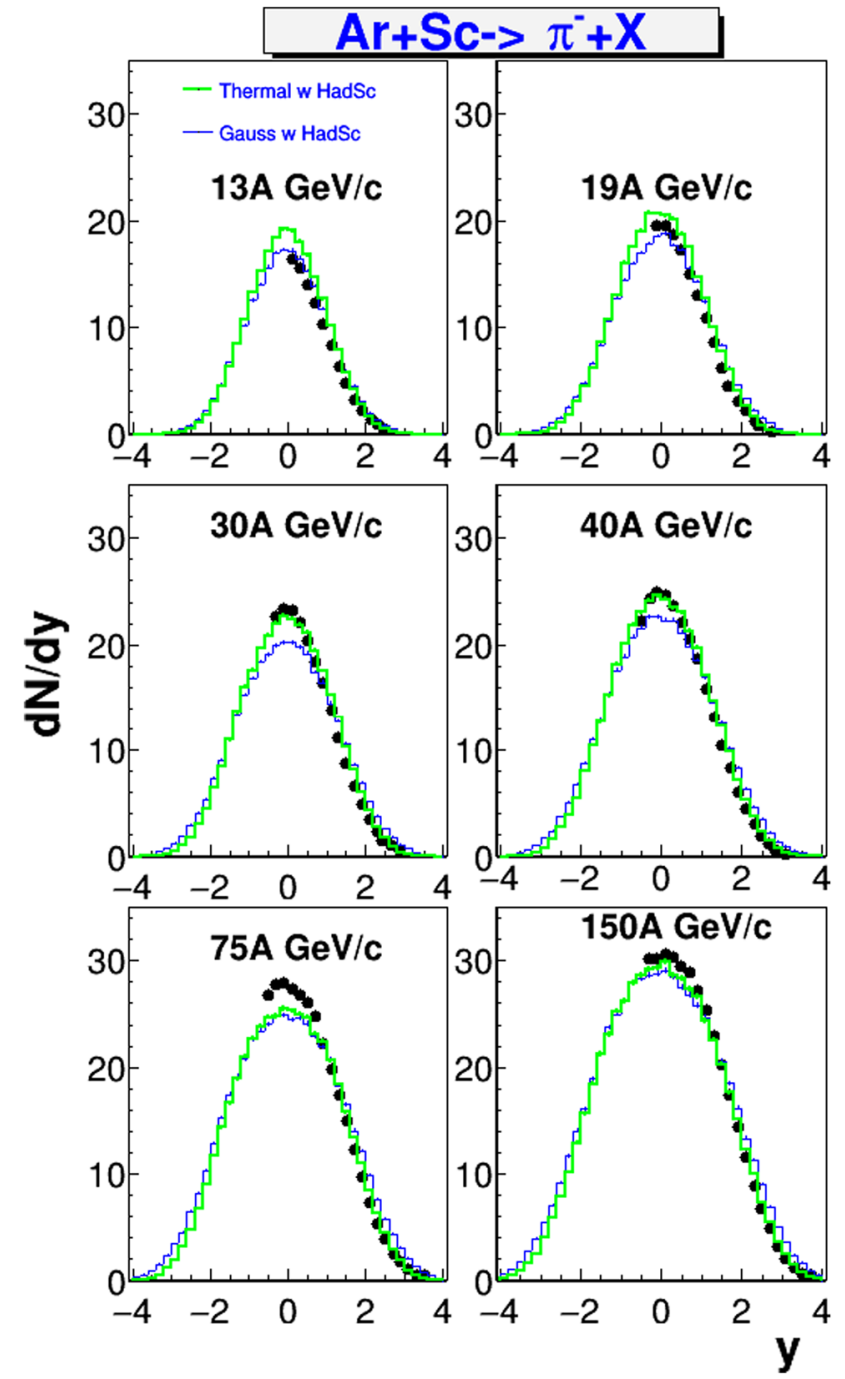

Fig. 9 Same as Fig. 8, but here the lines denote the Gaussian and thermal calculations with primary hadron rescatterings (HadRSc) included

$\pi^{-}$obtains larger $m_{\mathrm{T}}$ values due to expansion flow. It is interesting to notice that large deviation of thermal/HadRSc from Gaussian/HadRSc for larger $m_{\mathrm{T}}$ values appears at beam momentum of $30 \mathrm{~A} \mathrm{GeV} / \mathrm{c}$. This indicates that collective transverse flow appears in the 5\% most central $\mathrm{Ar}+\mathrm{Sc}$ collisions at about $30 \mathrm{~A} \mathrm{GeV} / \mathrm{c}$. It should be pointed out here that a rapid change of hadron production, such as sharp peak in the Kaon to pion ration, the start of a plateau in the inverse slope parameter for Kaons, and a steepining of the increases of pion production per wounded nucleon, are also observed in central $\mathrm{Pb}+\mathrm{Pb}$ collisions at $30 \mathrm{~A} \mathrm{GeV/c}$ by the NA49 experiment $[36,37]$.

A recent comparison of the measured rapidity distribution $(d N / d y)$ at NA61/SHINE data to UrQMD (version 3.4) [5], HIJING [6] and EPOS [16] models also show large discrepancies between the calculations and the measured $\pi^{-}$ $d N / d y$ in the $5 \%$ most central ${ }^{40} \mathrm{Ar}+{ }^{45} \mathrm{Sc}$ collisions at SPS 


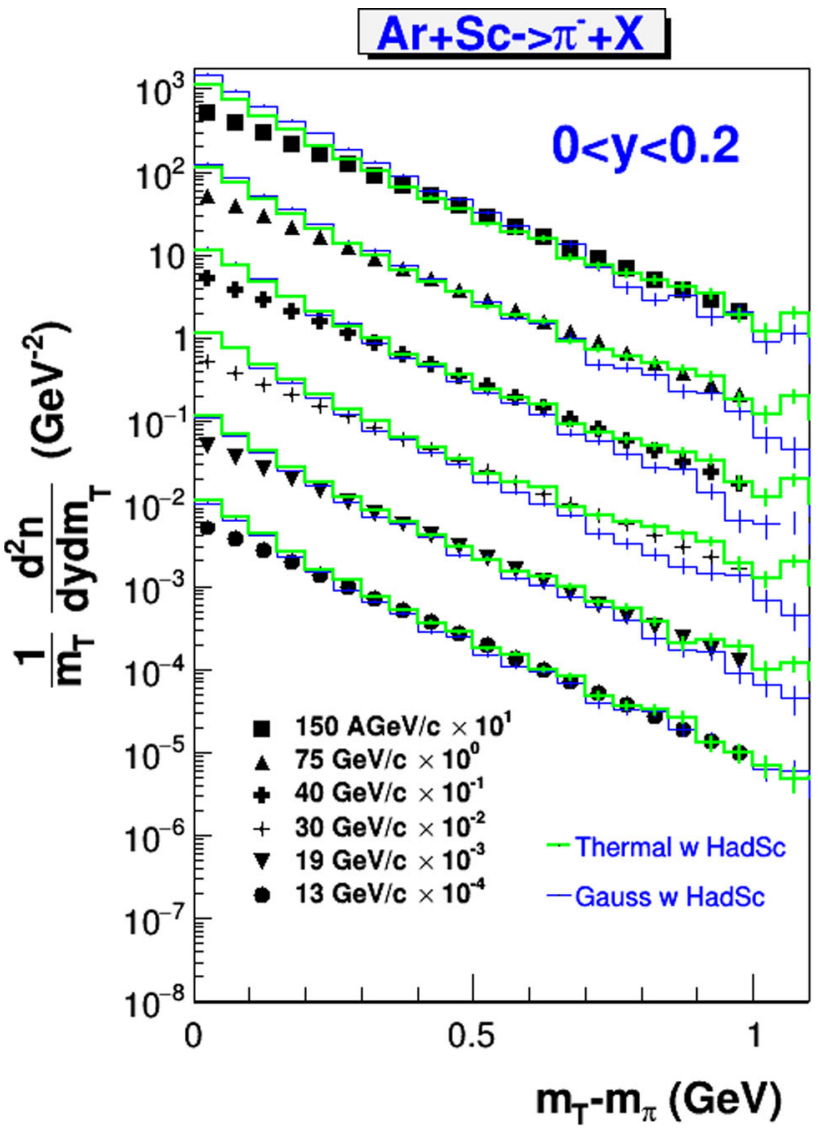

Fig. 10 The transverse mass spectra of $\pi^{-}$in the 5\% most central ${ }^{40} \mathrm{Ar}+{ }^{45} \mathrm{Sc}$ collisions at mid-rapidity $(0<y<0.2)$ for the whole SPS energies. The thin and thick lines denote the Gaussian and thermal models with primary hadron rescattering ( $\mathrm{HadSc}$ ), respectively. The closed points represent NA61/SHINE [9] data. For better readability, both the calculations and data points were scaled

energies [12]. The dependence of the inverse slope parameters of the $m_{\mathrm{T}}$-spectra as a function of center of mass energy for most central $\mathrm{Ar}+\mathrm{Sc}$ collisions are also studied. It was demonstrated that the EPOS model predictions are lower than the NA61/SHINE measurements. Predictions of UrQMD lie lower than measurements at low beam momenta and higher at high beam momenta. Finally, HIJING shows a concave behavior and unsatisfactory agreement with measurements.

\section{Summary and conclusions}

We have employed the Angantyr model, which extrapolates pp-dynamics, as described by PYTHIA 8.303 event generator, to study the production of $\pi^{-}$in central ${ }^{7} \mathrm{Be}+{ }^{9} \mathrm{Be}$ and ${ }^{40} \mathrm{Ar}+{ }^{45} \mathrm{Sc}$ collisions from beam momentum of $13 \mathrm{~A} \mathrm{GeV} / \mathrm{c}$ to $150 \mathrm{~A} \mathrm{GeV/c}$. First of all, the energy scale pace parameter of the multiparton interaction (MPI) settings has been tuned to pp-data. Angantyr predictions for pion production in central light(er)-ion beams are compared using Lund string and thermal models with tuned pp-parameters (see Table 1 of appendix). From the model calculations we can draw the following conclusions:

1. The transverse mass $\left(m_{\mathrm{T}}\right)$ spectra of pp-collisions at midrapidity $(0<y<0.2)$ are not correctly modelled at $m_{\mathrm{T}}-m_{\pi}>0.72 \mathrm{GeV}$, with Lund Gaussian $p_{\mathrm{T}}$ producing too few $\pi^{-}$particles.

2. The thermal model with temperature width of $T=0.31$ $\mathrm{GeV}$ is able to account for the transverse mass spectra of pp-collisions at large $m_{\mathrm{T}}-m_{\pi}$ values, where strong contributions from collective transverse flow are expected.

3. The Angantyr code using thermal model reproduces the stopping power of protons at mid-rapidity for the $20 \%$ most central ${ }^{7} \mathrm{Be}+{ }^{9} \mathrm{Be}$ collisions at different SPS energies.

4. Angantyr calculations employing thermal model are able to provide reasonable descriptions of the rapidity and transverse momentum distributions of $\pi^{-}$in the $20 \%$ most central ${ }^{7} \mathrm{Be}+{ }^{9} \mathrm{Be}$ collisions at CERN SPS energies.

5. Thermal model with simple primary hadron rescattering $(\mathrm{HadRSc})$ well explains the rapidity distributions and transverse mass spectra of $\pi^{-}$in the $5 \%$ most central ${ }^{40} \mathrm{Ar}$ $+{ }^{45} \mathrm{Sc}$ collisions at mid-rapidity for the whole SPS beam energies.

Thus, the main features of PYTHIA8 Angantyr event generator employing thermal model is the ability to reproduce the pion yield produced in inelastic pp- and light(er)-ion beam collisions at CERN SPS energies. However, both the thermal and Lund fragmentation models are not able to describe the transverse mass spectra of $\pi^{-}$for the reactions understudy at low $m_{\mathrm{T}}-m_{\pi}$ values. This discrepancy could be due to resonance decay effects, not included in the current study. The effects of realistic primary hadron rescattering and decays, which would require a detailed study of space-time picture, on hadron observables produced in light-ion beam collisions will be investigated in future publications.

Acknowledgements The author would like to thank the Deanship of Scientific Research at Umm Al-Qura University for supporting this work by Grant Code: (22UQU43312168DSR01).

Data Availability Statement This manuscript has no associated data or the data will not be deposited. [Authors' comment: The data appeared in this paper are available from the corresponding author upon request.]

Open Access This article is licensed under a Creative Commons Attribution 4.0 International License, which permits use, sharing, adaptation, distribution and reproduction in any medium or format, as long as you give appropriate credit to the original author(s) and the source, provide a link to the Creative Commons licence, and indicate if changes were made. The images or other third party material in this article are included in the article's Creative Commons licence, unless indicated otherwise in a credit line to the material. If material is not included in the article's Creative Commons licence and your intended 
Table 1 PYTHIA 8.303 parameters and their values for pp- and light(er)-ion collisions at SPS energies

\begin{tabular}{|c|c|c|c|}
\hline Parameter & Default PYTHIA8 & Gaussian model & Thermal model \\
\hline \multicolumn{4}{|l|}{ Reference energy scale } \\
\hline MultipartonInteractions: ecmRef & 7000 & 10 & 10 \\
\hline \multicolumn{4}{|l|}{ Energy rescale parameter } \\
\hline MultipartonInteractions: ecmPow & 0.215 & 0.16 & 0.16 \\
\hline \multicolumn{4}{|l|}{ String Lund fragmentation } \\
\hline StringPT: sigma & 0.335 & 0.31 & \\
\hline \multicolumn{4}{|l|}{ Thermal model } \\
\hline StringPT: thermalModel & Off & Off & On \\
\hline StringPT: temperature & & & 0.31 \\
\hline \multicolumn{4}{|l|}{ Hadron rescattering model } \\
\hline HadronLevel: Rescatter & Off & On & On \\
\hline Fragmentation:setVertices & Off & On & On \\
\hline PartonVertex: setVertex & Off & On & On \\
\hline
\end{tabular}

use is not permitted by statutory regulation or exceeds the permitted use, you will need to obtain permission directly from the copyright holder. To view a copy of this licence, visit http://creativecomm ons.org/licenses/by/4.0/.

Funded by SCOAP ${ }^{3}$.

\section{Appendix}

\subsection{Settings}

Table 1 gives a list of the settings that have been changed with respect to the default PYTHIA 8.303 tunes to obtain the results presented in Sect. 3. Non-listed parameters are set to the original Monash 2013 tunes of PYTHIA version 8.303 [30].

\section{References}

1. ALICE Collaboration, Nat. Phys. 13, 535 (2017)

2. CMS Collaboration, Phys. Lett. B 765, 193 (2017)

3. B. Alessandro et al., Eur. Phys. J. C 39, 335-345 (2005)

4. T. Sjöstrand, S. Mrenna, P. Skands, J. High Energy Phys., 2006, JHEP05 (2006). https://iopscience.iop.org/article/10.1088/ 1126-6708/2006/05/026 (2006)

5. S. Bass et al., Prog. Part. Nucl. Phys. 41, 255 (1998)

6. Xin-Nian. Wang, Miklos Gyulassy, Phys. Rev. D 44, 3501 (1991)

7. C.M. Zi-wei Lin, C.M. Ko, Phys. Rev. C 65, 034904 (2002)

8. A. Aduszkiewicz et al., (NA61/SHINE Collaboration), Eur. Phys. J. C 77, 671 (2017)

9. A. Aduszkiewicz et al., (NA61/SHINE Collaboration), Eur. Phys. J. C 74, 2794 (2014)

10. A. Acharya (NA61/SHINE Collaboration), Eur. Phys. J. C 81, 397 (2021)

11. A. Acharya, (NA61/SHINE Collaboration), Eur. Phys. J. C 80, 961 (2020)
12. A. Acharya, (NA61/SHINE Collaboration), Eur. Phys. J. C 81, 397 (2021)

13. B.I. Abelev et al., (STAR Collaboration), Science 328, 58 (2010)

14. J. Adam et al., ALICE Collaboration. Phys. Lett. B 754, 360 (2016)

15. K. Werner, Phys. Rev. Lett. 98, 152301 (2007)

16. K. Werner, Nucl. Phys. B Proc. Suppl. 175-176, 81-87 (2008)

17. Christian Bierlich, Gösta. Gustafson, Leif Lönnblad, Harsh Shah, JHEP 10, 134 (2018)

18. T. Sjöstrand et al., Comput. Phys. Commun. 191, 159-177 (2015)

19. Torbjörn Sjöstrand, Marius Utheim, Eur. Phys. J. C 80, 907 (2020)

20. B. Andersson, G. Gustafson, B. Nilsson-Almqvist, Nucl. Phys. B 281, 289 (1987)

21. B. Nilsson-Almqvist, E. Stenlund, Comput. Phys. Commun. 43, 387 (1987)

22. Torbjörn Sjöstrand, Comput. Phys. Commun. 82, 74 (1994)

23. Nadine Fischer, Torbjörn Sjöstrand, J. High Energy Phys. 2017, 140 (2017)

24. A. Motornenko, V.V. Begun, V. Vovchenko, M.I. Gorenstein, H. Stoecker, Phys. Rev. C 99(3), 034909 (2019)

25. Wojciech Broniowski, Maciej Rybczyński, Piotr Bożek, Comput. Phys. Commun. 180, 69 (2009)

26. A. Bialas, M. Bleszynski, W. Czyz, Nucl. Phys. B 111, 461 (1976)

27. H.J. Drescher, M. Hladik, S. Ostapchenko, T. Pierog, K. Werner, Phys. Rep. 350, 93 (2001)

28. B. Andersson, G. Gustafson, G. Ingelman, T. Sjöstrand, Phys. Rep. 97, 31-145 (1987)

29. B. Andersson, G. Gustafson, B. Söderberg, Z. Phys. C 20, 317 (1983)

30. P. Skands, S. Carrazza, J. Rojo, Eur. Phys. J. C 74, 3024 (2014)

31. A. Donnachie, P.V. Landshoff, Phys. Lett. B 296, 227-232 (1992)

32. Christine O. Rasmussen, Torbjörn Sjöstrand, Eur. Phys. J. C 78, 461 (2018)

33. M.M. Aggarwal et al., (WA98 Collaboration), Phys. Rev. C 83, 926 (1999)

34. W. Cassing, E.L. Bratkovskaya, Phys. Rev. C 78, 034919 (2008)

35. J. Mohs, S. Ryu, H. Elfner, J. Phys. G Nucl. Part. Phys. 47, 065101 (2020). (and reference therein)

36. S.V. Afanasiev et al., Phys. Rev. C 66, 054902 (2002)

37. C. Alt et al., Phys. Rev. C 77, 024903 (2008) 